\title{
De la lectura de biografías a la escritura crítica en prácticas profesionales en la escuela'
}

\author{
From Reading Biographies to Critical Writing In-Service \\ Training at School
}

\author{
ORLANDO ALBERTERIS GALBÁN \\ VIVIANA CAÑIZARES HINOJOSA \\ ORLANDO RODRÍGUEZ DÍAZ
}

Universidad Ignacio Agramonte Loynaz

\section{Cuba}

orlando.alberteris@reduc.edu.cu

viviana.canizares@reduc.edu.cu

orlando.drodriguez@reduc.edu.cu

(Recibido: O4-II-202O; aceptado: $2 \mathrm{I}-\mathrm{O} 5-2 \mathrm{O} 2 \mathrm{I}$ )

Resumen. El presente trabajo tiene como propósito principal analizar la relación entre los niveles de comprensión lectora de estudiantes de la carrera Lenguas Extranjeras y su competencia escrita a partir de biografías de personalidades cuyos nombres llevan la escuela donde realizan su práctica profesional. Para ello se ha empleado un estudio descriptivointerpretativo a partir de la implementación de siete tipos de acciones ( 3 de lectura y 4 de escritura), en una muestra de 20 estudiantes de tercer año de la carrera Lenguas Extranjeras de la Universidad de Camagüey durante el curso 2018-2019. Los resultados mostraron dos hallazgos fundamentales: el primero, los bajos niveles de competencia lectora en textos de género biografía, fundamentalmente en la identificación de comportamientos de los personajes y sus causas, así como en la identificación de la organización hermenéutica del texto; el segundo, en el nivel alcanzado en la escritura argumentada incidió significativamente la baja competencia lectora de los estudiantes y la carencia de argumentos propios. La interconexión lectura-escritura constituyó un factor de relevancia para el mejoramiento de la didáctica de la lectoescritura en la carrera.

Palabras clave: comprensión lectora; competencia escrita; textos biográficos; acciones de lectura y escritura; práctica laboral.

\footnotetext{
${ }^{\text {I }}$ Para citar este artículo: Albertereis Galbán, Orlando; Cañizares Hinojosa, Viviana y Rodríguez Díaz, Orlando (2O22). De la lectura de bibliografías a la escritura cítrica en prácticas profesionales en la escuela. Alabe 25 . [www.revistaalabe.com] DOI: I0.15645/Alabe2022.25.I
} 
Abstract. The present paper deals with the analysis of the relation between reading comprehension in students of the English Foreign Language major, and their writing competence from biographical texts of the school of the same name, where the students do their in-service training. To this end, a descriptive-interpretative study was applied, and seven types of actions ( 3 reading actions and 4 writing actions) were implemented to a sample of 20 thirdyear students of the English Foreign Language major in Camagüey University during the 20182019 academic year. The results showed two main findings: first, the low levels in reading competence in biographical texts, mainly in identifying character behavior and its causes, and likewise the hermeneutic organization of the text; second, the argumentative writing was significantly affected by the low levels in students' reading competence and a lack of their own arguments. The interconnection between reading-writing constitutes a relevant factor for the improvement of the didactics of reading and writing in the major.

Keywords: reading comprehension; writing competence; biographical texts; reading and writing actions; professional practice. 


\section{Introducción}

La biografía de figuras y personalidades de la ciencia, la historia, la pedagogía, la literatura, etc., ha sido utilizada en la práctica pedagógica de lenguas extranjeras como un elemento didáctico que ayuda a los estudiantes no solo en el uso de la información escrita como un medio de comunicación, sino también como medio para el desarrollo de la interacción dialógica con el texto biográfico, para el acceso a formas de narración, procedimientos lingüístico-discursivos, entre otros aspectos que conducen a un mejoramiento de las competencias de lectoescritura de los estudiantes.

La biografía en tanto práctica lingüística, ocupa un lugar importante en el desarollo de la competencia comunicativa de los estudiantes y forma parte de las acciones y mecanismos comunicativos que como género textual moviliza y presupone. De ahí que la biografía como práctica, se instala en la didáctica de las lenguas extranjeras como recurso para fijar "significaciones sociales complejas que conciernen a las actividades de aprendizaje lingüísticas” (Dolz y Gagnon, 20IO: 508). La biografía constituye una circunstancia para el ejercicio de lectura crítica y valoraciones sobre sí mismo y los otros, para la expresión de significados de diversa índole, aspectos que ejercen una indiscutible influencia en la afectividad del estudiante como un indicador de su actuación y proyecto de vida dentro de la esfera personal y profesional. Como tipo de escritura, la biografía incorpora componentes cognitivos, sociales y textuales e integra, según Grabe y Kaplan (I998), tres aspectos importantes: el procesamiento cognitivo del escritor, los recursos lingüísticos y textuales que participan de la tarea de escritura y los factores contextuales que fuertemente moldean la naturaleza de la escritura.

La biografía, frecuentemente como tipo de narración, revela actos e implicaciones de las personas en referencia al contexto social, cultural y político de la época. De esta forma intenta reconstruir el pensamiento narrativo de la persona, el cual incluye las intenciones de sus acciones, vicisitudes y consecuencias (Montealegre, 2004: 25I). Como texto narrativo, la biografía tiene efectos en el lector a través de los significados, los cuales, según Bruner (I995), dependen del empleo que realiza el lector de una serie de instrumentos de su cultura (lenguaje, su experiencia anterior, sus conocimientos previos y sus modos de representación mental, etc.), previamente adquiridos que guían su interpretación del texto. Van Dijk y Kintsch (I983) proponen que en la comprensión de un texto participan tanto la construcción de una representación del significado de dicho texto, como la construcción de una representación mental de la situación descrita en él. Para Kintsch (1998), la comprensión profunda requeriría necesariamente la construcción de un modelo de la situación descrita en el texto, lo que requiere de la interacción entre la representación construida del texto y el conocimiento almacenado en la memoria de largo plazo del lector.

Desde esta perspectiva, la biografía se constituye en una actividad de comprensión y producción de sentido; no es un simple trabajo de decodificación sino un "proceso de interrogación, participación y actualización por parte de un receptor activo que 
la reconoce como un proceso de cooperación textual" (Santiago, Castillo y Luz, 2007: 28). La biografía como tipo de escritura que orienta la práctica de lectura, se enmarca en la instancia interpretativa como tipo particular de texto, e implica un "desplazamiento" interpretativo, un "ponerse en marcha" desde diferentes niveles de interpretación. La lectura en este sentido "es un ritual iniciático de búsqueda y encuentro para aproximarse a una verdad, a lo que está entre las palabras" (Hamui, 2OI4: I32).

Por consiguiente, en este trabajo presentamos los resultados de una experiencia investigativa en curso sobre prácticas de lectura y escritura críticas de biografías, como parte de un proyecto de práctica profesional bajo el título "El nombre de la escuela donde realizo mi práctica" "The school's martyr's name or personality, where I do my in-service training”).

Se plantea como objetivo general de este trabajo mostrar una visión didáctica sobre el uso del género biografía como recurso para la lectura crítica, la interpretación y la escritura argumentada en inglés. Así, este trabajo se propone analizar el proceso de los estudiantes al leer críticamente biografías para identificar argumentos y supuestos, reconocer relaciones e interrelaciones importantes, significados subyacentes y escribir sobre posiciones y dilemas morales presentes en esos textos, construir significados propios, crear posturas interpretativas y valorativas, como incentivo para el desarrollo de competencias en lengua extranjera. Por otra parte, este trabajo tiene un interés pedagógico que consiste en la comprensión del modo en que la didáctica de lenguas extranjeras ha de concebir y guiar la lectura y la escritura.

Como objetivos específicos del presente estudio se plantean los siguientes:

I. Leer de forma selectiva, analizar y sintetizar información en textos biográficos.

2. Realizar el análisis lingüístico-discursivo del género biografía.

3. Reconocer la existencia de interconexiones entre la lectura y la escritura durante el proceso de deconstrucción del género biografía.

4. Buscar información en otras fuentes a fin de desarrollar la intertextualidad.

5. Realizar una escritura argumentada.

Desde la perspectiva del docente se plantean dos objetivos fundamentales: I. Posibilitar la reflexión sobre experiencias y expectativas profesionales, así como la valoración de dilemas morales presentes en los textos biográficos; 2 . Evaluar los efectos de las acciones aplicadas con el fin de crear criterios metodológicos que orienten la actuación del profesor en el trabajo de lectoescritura en el aula.

\section{La biografía como narración}

La biografía como narración es un recurso de transmisión de la cultura y es la base para conformar percepciones sobre el mundo. Según Bruner (1994, 1997. Citado en Montealegre (2004), la narración, más que una forma de representación, es "un instrumento de la mente en la construcción de la realidad" y posee una serie de características 
que, desde nuestro punto de vista, aportan valiosos elementos a la didáctica comunicativa de las lenguas extranjeras. Estas características son resumidas por Montealegre (2004: 252):

I. La diacronicidad: Todo evento de una narración sucede a través del tiempo, y está determinado de forma subjetiva en donde cada sujeto o personaje tiene un propio compás. En concreto, cada narración presenta formas especiales de temporalidad.

2. La particularidad: Las narraciones hacen referencia a hechos particulares pero escritos en forma genérica.

3. El estado intencional: Los sucesos y el ambiente en el cual están inmersos los personajes, se relacionan con sus estados intencionales (deseos, creencias, valores, etc.); a partir de éstos se pueden deducir las razones por las cuales suceden las cosas, más no sus causas estrictas.

4. La organización hermenéutica: El arte de la interpretación de textos o hermenéutica no se logra, para Bruner, de manera racional o empírica sino de modo intuitivo, pues no se trata de encontrar la veracidad en el texto sino de reconstruir su significado, de interpretar la historia mediante el análisis de la interdependencia que existe entre las partes y el todo. El todo constituye el sentido de la historia y las partes las funciones subordinadas al sentido.

La narración, desde nuestro punto de vista, aporta valiosos elementos a la didáctica comunicativa de las lenguas extranjeras. Así, la narración cumple con ciertas convenciones discursivas e involucra rasgos lingüísticos de temporalidad cuyo tratamiento en el aula de idioma es esencial por su aporte a la competencia lingüística. Por ejemplo, el empleo de organizadores temporales, sucesión, simultaneidad, causalidad, etc. El estado intencional de la narración posibilita el trabajo con posiciones críticas en la lectura y el ejercicio de expresión de opiniones y juicios, de causas y efectos, de comparación, de hipótesis, así como el descubrimiento de connotaciones en la psicología de una figura determinada, valores dentro del contexto o visión subjetiva subyacente del autor, de delimitación de elementos de la etopeya (aunque en ocasiones no se revelan de forma explícita el carácter, acciones y costumbres de la persona, es posible hacer asociaciones diversas a sentimientos internos de ella). Este es un aspecto de vital importancia en la lectura de biografías, lo que conduce a dar importancia a aspectos específicos dentro de la narración que hacen referencia a la particularidad de hechos y personalidades.

De todo ello es posible asumir la biografía como medio de reconstrucción de significados, de interpretación mediante el análisis del todo y sus partes. Esta postura hermenéutica posibilita el reconocimiento del todo y las partes en el contexto de la biografía y el descubrimiento de las cualidades significativas a partir del examen de la forma en que han sido organizadas las partes del todo. Hernández (2OI2: 29) apunta que "las relaciones de parcialidad (el todo y las partes) revelan el sentido del detalle, intentan determinar cómo la parte puede revelar el significado del todo. Es preciso delimitar las 
partes manifiestas y las partes implícitas; las esenciales y no importantes, y los diferentes partes desde los cuales puede verse el todo".

Un elemento fundamental en la didáctica de lenguas extranjeras postula la capacidad del estudiante de penetrar en el mundo de las relaciones y de estimularlo a hacer conexiones entre las partes y el todo (Arizpe \& Styles, 20I2), a penetrar en lo más sutil, más imaginativo o más creativo, a valorar los significados que se expresan en el texto biográfico, acción que no solo se expresa en el reconocimiento del valor de la biografía en sí, sino también en la forma en que se conjugan los artificios lingüísticos para hacerla valer.

En este sentido es importante acotar que la propia valoración se convierte en otro modo de crear, lo que para la enseñanza y aprendizaje de lenguas extranjeras es un elemento crucial. No obstante, ello supone un apropiado nivel de competencia lectora y de intertexto personal, es decir, de saberes intertextuales, que permiten relacionar distintos textos y diferentes manifestaciones culturales entre sí (Sánchez, 2OII), todo lo cual conduce a interrogar ¿Qué relaciones se establecen? ¿Por qué se seleccionan esas partes? ¿Qué intención de significados subyace en la selección? ¿Cuáles son los referentes? ¿Qué sentido aportan? Los referentes son elementos a los que el texto hace referencia y forman parte de la trama sensitiva de la conciencia del autor. Al captar estos referentes, el lector comienza a establecer relaciones entre ellos y asimila así de forma generalizada aquella parte de la herencia histórico - cultural de la humanidad que el autor del mismo nos muestra. Por su parte, los sentidos no son más que los significados que le asigna el lector a los propios significados que aparecen en el texto.

Desde esta perspectiva la biografía se postula como una interacción dialógica, en tanto posibilita formular opiniones y adoptar posturas o posiciones frente a diversos hechos o acontecimientos en los que la persona se ha visto involucrada. En este sentido, el hecho de abordar posicionamientos permite examinar uno de los procesos más complejos y fundamentales en la enseñanza de lenguas, es decir, el relacionado con la evaluación de perspectivas ajenas y la asunción de posturas, puntos de vistas, creencias, sentimientos o emociones. Como se ve, se trata de opciones que aluden a la relación pensamientolenguaje y a la interrelación de lo cognitivo y lo afectivo del proceso de enseñanza aprendizaje de lenguas extranjeras.

En esta línea de pensamiento, Hamui (20I4: I32) acota que una "interpretación motiva la movilidad del texto, la deconstrucción, la derivación, la elección, la relación con otros textos, objetos y motivos; implica ecos del pasado, vínculos con el presente, afinidades con la vida, la historia”. Este autor considera que la lectura es un acto de re-escritura, es decir, se crea un texto "nuevo" en el momento de interpretar. La deconstrucción se refiere a un desmontaje textual, considerado como "una estrategia intencional que aplicamos a la narración al detenernos en su lenguaje y su sentido".

La biografía, por tanto, constituye un género muy ligado a las actividades académicas y de la práctica profesional. La biografía es un recurso importante para dar sentido a la propia experiencia personal del estudiante, para hacerlo consciente de su rol social y 
profesional, para contribuir a su desarrollo comunicativo en la lengua extranjera. Desde este punto de vista el trabajo con este género tiene fuertes implicaciones en el desarrollo integral del estudiante:

- El conocimiento de la vida y obra de grandes personalidades constituye un pilar de su interpretación y reflexión de la actividad profesional que realiza. Con ello se posibilita "conocer y ser consciente de los argumentos teórico-prácticos que sustentan su práctica educativa, analizar las claves y los referentes que han moldeado el pensamiento y actuación del mismo, reorientar la actuación profesional apoyándose en una propuesta de trabajo" (Sepúlveda y Rivas, 2003: $380)$.

- La biografía permite repensar en las experiencias propias y en la construcción de la propia identidad; posibilita reflexionar sobre las experiencias personales y profesionales vividas, evaluar su propio proceso de aprendizaje y sus expectativas profesionales actuales y futuras.

- Posibilita la idea de pensar en el fenómeno educativo desde un punto de vista creativo y constructivo (Aiello, Iriarte, \& Sassi, 20II. Citados por Bazán, Ferrari, Castro, Delgado y Hernández, 20I6).

- Contribuye al mejoramiento de las competencias de lectoescritura de los estudiantes, así como a la inserción en prácticas socializadas colaborativas.

De todo ello se asume que la biografía además de contribuir al desarrollo de la competencia comunicativa, ejerce una indiscutible influencia en la afectividad del estudiante como un indicador de su actuación y proyecto de vida dentro de la esfera personal y profesional.

\section{Metodología}

El presente trabajo tiene una base descriptiva-interpretativa, con orientación al análisis del proceso de lectura crítica de textos biográficos y de construcción escrita de opiniones o posicionamiento frente a ideas seleccionadas de estos textos. La muestra fue de 20 estudiantes de tercer año de la carrera Lenguas Extranjeras de la Universidad de Camagüey "Ignacio Agramonte", durante el periodo de práctica profesional en la escuela, con una extensión de I9o horas correspondientes a la Práctica Laboral Investigativa del plan del proceso docente. Se caracterizó por el desarrollo de actividades de lectoescritura acerca de las biografías de las personalidades cuyo nombre lleva la escuela donde se realizan las prácticas profesionales. Con este fin los estudiantes debían realizar acciones lectoras de biografías y a partir de ellas debían realizar producciones escritas de opiniones o de toma de posición frente a dilemas morales o comportamientos de las personalidades implicadas en las biografías.

Las actividades de la lectura de biografías se enfocaron desde la perspectiva narrativa, tomando los criterios expuestos por Bruner (I994), y aspectos de la lectura y 
escritura crítica desarrollados por Serrano y Madrid (2007), en particular sobre la reconstrucción del significado, intencionalidades e ideologías implícitas en los discursos, así como aspectos concernientes al análisis de los géneros escritos (Cassany, 20o6). Se asumen además criterios expuestos por Avendaño de Barón (20I6) sobre la reflexión y evaluación crítica argumentada del texto (pensar sobre el texto leído, emitir juicios de valor y asumir posturas razonadas).

Para el abordaje del género biografía se asumieron los siguientes criterios: a) los géneros discursivos ofrecen procedimientos lingüísticos para identificar determinadas características léxico-gramaticales y retóricas-estructurales, las cuales permiten modelar las prácticas comunicativas de los estudiantes (Jarpa, 2OI3); b) el análisis del género debe considerar los aspectos socioculturales y contextuales en los cuales se incluyan los elementos básicos del acto comunicativo, aspectos discursivos referidos a la organización del discurso, aspectos gramaticales y léxicos que consideran la elección de las palabras y estructuras (Cassany, 20o6); c) el género abarca tanto cuestiones formales como de contenido, es decir, qué se dice, cómo, por qué y para qué (Cassany, 2008).

Desde la perspectiva metodológica de Cassany (2008) se asumió que aprender a leer y escribir un género discursivo es aprender a emplearlo en la vida real y, también, a dominar la parte de la actividad que se desarrolla con este género, relacionando los contenidos lingüísticos con ejemplos de cada género y con tareas prácticas de lectura y escritura de los mismos.

Teniendo en cuenta los aspectos descritos hicimos una propuesta de acciones para la lectura y escritura del texto biográfico como muestra del género en cuestión. Para la lectura del texto biográfico se enfatizaron las siguientes acciones: I) Identificación de formas especiales de temporalidad, rasgos lingüísticos de temporalidad (empleo de organizadores temporales, estructuras cronológicas, simultaneidad, causalidad, formas adverbiales de tiempo); 2) Identificación de la psicología de la persona, carácter, valores, acciones, costumbres y comportamientos, así como las razones a esos comportamientos; 3) La organización hermenéutica del texto, el análisis de la interdependencia que existe entre las partes y el todo, el descubrimiento de las cualidades significativas a partir del examen de la forma en que han sido organizadas las partes del todo, la valoración de los significados que se expresan en el texto biográfico y la forma en que se conjugan los artificios lingüísticos para hacerlos valer.

Para la escritura se tuvieron en cuenta las siguientes acciones: 4) Estructuración de las ideas en secuencia lógica, en correspondencia con las exigencias sintácticas (cohesión) y semánticas (coherencia); 5) Uso adecuado de conectores, así como la intertextualidad; 6) Adecuación ortográfica (spelling, Punctuation, and Mechanics) correcta para mantener el significado y expresarlo con claridad, pertinencia y adecuación a la situación comunicativa; 7) Posicionamiento crítico argumentado frente al texto y presentación de posiciones propias. 


\section{La experiencia}

Las actividades realizadas tuvieron la siguiente secuencia. Primero, se trabajó con las biografías de cuatro figuras cuyos nombres llevan las escuelas seleccionadas. Se tuvo en cuenta en qué escuela fueron insertados los estudiantes durante la práctica profesional. Así, quedaron establecidas cuatro escuelas con cinco estudiantes insertados. Corresponde, entonces, cuatro biografías ("Inés Luaces”, "Ana Betancourt”, "Ignacio Agramonte", "La Avellaneda").

Durante la primera semana de práctica, los propios estudiantes buscaron las biografías correspondientes a cada figura. Después se realizó una primera sesión de trabajo en el aula de idioma con el fin de poder orientar los aspectos necesarios a tener en cuenta, así como para crear el contexto específico de aprendizaje. Asimismo, se promovió una discusión oral sobre el género biográfico, su estructura, aspectos lingüísticos y discursivos característicos, así como sobre la estructura (cronológica o narrativa) del texto biográfico.

Después de una semana de práctica se realizó la segunda sesión de trabajo en el aula de idioma para revisar las respuestas de los estudiantes de las actividades de lectura descritas anteriormente, así como para corregir imprecisiones en el orden lingüístico y cultural. Esta sesión de trabajo permitió explorar el conocimiento previo de cada estudiante y su posición inicial frente al texto biográfico. Se procedió, además, a realizar una lectura selectiva de aspectos cruciales que identificaban a la figura, principales acontecimientos o eventos en los que estuvo involucrada, así como dilemas morales, comportamientos, creencias, etc. Se orientó de manera guiada la identificación de los conceptos e ideas claves dentro del texto. Como esta actividad inicialmente resultó difícil para los estudiantes, el profesor les proporcionó acompañamiento y ayuda pedagógica. Seguidamente se asignó la búsqueda de otros textos relacionados con el tema o sobre estas ideas o conceptos claves.

Estas actividades de deconstrucción del género tuvo como propósito "introducir a los estudiantes en un ejemplo de texto perteneciente al género en estudio" (Moyano, 2007: 578), de manera que los estudiantes pudieran entender cuál era la organización del texto en relación a la producción de significados y el cumplimiento del propósito social y comunicativo perseguido, las condiciones del contexto y los elementos de la situación retórica que condicionan las elecciones lingüísticas en el texto.

Posteriormente se orientaron las actividades de escritura, las cuales tuvieron un mayor tiempo en el cronograma de realización y corrección (dos semanas). Estas actividades tuvieron que ver fundamentalmente con la escritura argumentada sobre los dilemas morales y la toma de posición sobre ellos. Se hizo énfasis en cómo lograr la construcción por escrito de una posición a partir del análisis, la interpretación y evaluación de las ideas claves seleccionadas.

A partir de aquí los escritos de los estudiantes fueron sometidos a revisión de forma individual, adecuación y retorno permanente a operaciones de textualización y revi- 
sión, en relación con el manejo de argumentos para justificar su posición y convencer, así como con las exigencias sintácticas y semánticas del escrito, el uso de conectores y procedimientos de argumentación, tales como de asociación, comparación, analogía, ejemplificación, empleo de criterios de autoridad (citación), presentación de evidencias, justificación con base en creencias, vivencias personales, testimonios, etc. Esta etapa de construcción escrita tuvo como propósito también la comparación con otras fuentes para darle mayor objetividad y corrección al escrito. Así, se reajustaron aquellos elementos dispersos acordes a los requerimientos y exigencias consensuadas. También se logró la necesaria retroalimentación en el sentido de contrastar información y afianzar formas discursivas apropiadas.

Esta etapa de construcción del escrito exigió de los estudiantes la aplicación de los elementos tratados en la etapa de deconstrucción, teniendo en cuenta las particularidades del género de estudio, así como la reflexión acerca de los recursos para la producción de sentido. Para esta etapa se trabajó con un modelo de procedimientos para introducir el género (Tabla I).

Tabla ı. Modelo de procedimientos para introducir el género biografía.

Situación de comunicación: Los estudiantes se convierten en emisores y a la vez en destinatarios (receptores) que socializan tareas comunicativas de manera formal sobre personalidades históricas, literarias, de la pedagogía, del arte.

Objetivos: Relatar por escrito las experiencias de la vida y obra de una destacada figura de la historia, de la literatura o del arte... (El nombre del mártir cuyo nombre lleva la escuela donde realizo mi práctica laboral) y enjuiciar dilemas morales o posturas de esas figuras.

Planificación: 1. Introducción de la biografía. 2. Presentación y relato de las experiencias de la vida y obra de la figura histórica (estructura narrativa típica de la biografía/ cronología de hechos). 3. Cierre de la biografía.

Unidades lingüísticas: Léxico para describir y situar las acciones, recursos estilísticos y fórmulas para interesar al lector, procedimientos lingüísticos típicos de la narración, empleo de organizadores temporales, expresiones para situar los eventos, empleo de recursos para expresar sucesión, simultaneidad, causalidad, así como para dar argumentos.

Veamos dos ejemplos de extractos de los relatos de los estudiantes en esta etapa de construcción:

\section{Ejemplo I.}


Ignacio Agramonte y Loynáz:

I am a student of foreign language (English major) career and I do my re-service training at "Ignacio Agramonte" school. Why is my school named in honor of Ignacio Agramonte? The school is named after the well-known Cuban patriot Ignacio Agramonte y Loynáz. He was a Cuban revolutionary, who played an important part in the Cuban war for independence. He was born in Puerto Príncipe, Camagüey, Cuba, in December 23, 1841.

He became Major General of the Cuban forces for the military district of the province of Camaguiey, where he organized the best cavalry troops in the Cuban Army.

Why did he gain everyone's respect and love? The commander of his Cavalry Corps, Henry Reeve, named him "El Mayor", implying Agramonte was the best of all the $\mathrm{Cu}$ ban Major Generals. He was also known as "El Bayardo", implying gallantry, patriotism and courage.

José Martí, in his beautiful patriotic text "Céspedes y Agramonte", recognized the magnanimity of two men who offered their life to Cuban independence. He wrote: "Of Céspedes the vigor, of Agramonte the virtue. Agramonte, as Martí described him, was a "diamante con alma de beso".

On the centenary of Agramonte's death, Silvio Rodríguez premiered his song El Mayor, in honor of the fallen hero. He described this fallen hero with these words: "mortales ingredientes armaron al Mayor: luz de terratenientes y de Revolución", "la vergüenza, el amor".

Agramonte was a man of virtue, courage, and his loving character. His virtue means moral rectitude. His courage describes his spirit that enables him to face danger and vicissitudes with confidence and resolution. His loving character is an example of deep affection toward a person, such as that of arising from his beloved Amalia.

There are four things I admire about Agramonte. First, his love for his wife. Second, his military achievements during the war time. One of his best known achievements was the rescue of Sanguily, which was considered by Commander in Chief Fidel Castro as an event with a great historical meaning. This event has passed to history as one of most extraordinary military actions, an event that raised the spirits in the Cuban field in difficult moments. It was without a doubt one of the greatest brave deeds that were written in our battles for independence.

The third thing I admire the most about Agramonte is his courage, the quality to face danger and vicissitudes with resolution. One who reads his short life-time history can realize this quality. The fourth thing has to deal with his sense of shame. He hated dishonor. Once Agramonte, in camp at La Redonda, a delegation asks Major Agramonte, "with what will you continue this bloody fight?" His famous response: "With the shame of the Cuban People!” (“;con la vergüenza!”).

En el escrito del primer ejemplo es evidente que el estudiante ha tratado de utilizar algunos recursos adquiridos durante la etapa de deconstrucción. Ejemplo de esos recursos son la interrogación para interesar al lector (Why is my school...), la ejemplificación (An example...), la temporalidad (during), la adición (moreover), la causalidad (because of...), estructuras de enumeración (first, second, third), giro idiomático, (in spi- 
te of...), continuidad (in fact- en realidad, en verdad), oposición (although), argumentos basados en fuente o autoridad (...was considered by Commander in Chief...; José Martí recognized ...), pronombres sustitutivos (he), de referencia (it), argumentos por adhesión u opinión (I admire...), exclamación retórica (jcon la vergüenza!), definición por sinónimo (His virtue means moral rectitude; his loving character is an example... ), analogía como recurso narrativo (...the magnanimity of two men...), uso de la metáfora (diamante con alma de beso).

\section{Ejemplo 2.}

Ana Betancourt de Mora:

Spain. She also is known by her support of women's emancipation as part of this struggle".

"She was born in December 14, 1832, in Camaguey, Cuba".

"According to an annotated biography, in Cuban and Cuban-American Women, written by Stoner, K. Lynn (2000), Ana Betancourt was one of the first to argue for women in Cuba. In 1869, she addressed the Constitutional Assembly of Cuban patriots at Guáimaro in which she linked female emancipation to the abolition of slavery and the struggle against colonialism".

"Because of her continuing struggle against colonialism and for women's emancipation she was sentenced to exile in Spain where she lived the rest of her life. However, she continued to support the cause of Cuban independence from her exile".

"Ana Betancourt died at the age of 69 in 1901". In 1979 was officially sanctioned the state award "Ana Betancourt", the highest award of the Federation of Cuban Women". She carried out many significant patriotic actions. In 1872 visited the Presidents of the USA, Ulises Grant. She asked for his interceding for the reprieve of the students of medicine, taken prisoners in the events of November 1871. In Spain, in 1889 she began to copy the Agramonte's diary that was in hand of the Spanish general Juan Ampudia.

"I consider that Ana Betancourt was an example of Cuban women who offered her life to independence and to women's rights. Therefore, there are some characteristics of Ana Betancourt that I admire. I admire her tenacity and her total devotion to independence and women's fairness. She never regretted her decision to participate in revolutionary actions that were taken. Her entire life was full of revolutionary experiences that made her stronger and molded in with a spirit of a woman with resolution in purpose and action".

En el escrito del segundo ejemplo también el estudiante ha tratado de utilizar algunos recursos adquiridos durante la etapa de deconstrucción, aunque en menor medida. Así, por ejemplo se hace explícito el uso de diferentes tipos de conectores, estructuras pronominales, cronológicas y de temporalidad, la causalidad, estructuras comparativas adjetivas (that made her stronger), argumento por opinión (I consider) y por grado de acercamiento o distanciamiento con respecto al contenido citado (according to...). 
Como se observa en ambos escritos, aún el desarrollo de recursos lingüísticodiscursivos en los estudiantes es incipiente. Asimismo, el manejo de recursos retóricos del género, la progresión temática y desarrollo del contenido de las biografías, así como la realización gramatical todavía se encuentran en un nivel muy bajo. No obstante, la experiencia mostró algunos logros en la escritura del género biografía que son indicadores de desarrollo de la competencia escritural de los estudiantes en lengua extranjera.

La mención de características específicas de la persona y los eventos en los que estuvo involucrada hacen más específica la vinculación entre partes del texto biográfico, como recurso de cohesión con elementos léxicos o cadenas léxicas que contribuyen a la construcción de los campos semánticos del texto. La estructura retórica de los escritos se construyó utilizando la inserción de voces ajenas para dar cuenta de determinados sucesos en la vida de las personas, así como el uso de ciertas formas que aseguraron las relaciones lógico-semánticas entre las proposiciones de los textos, tales como repeticiones, pronombres, sinónimos, entre otras. No obstante, se evidenció que muchas de las ideas expuestas fueron extensiones de otras provenientes de diversas fuentes, lo que indicó un nivel bajo de creatividad y postura personal por parte de los estudiantes.

\section{Resultados}

A fin de constatar los resultados específicos en la lectura de textos biográficos y la competencia escritural de los estudiantes sobre la lectura, se han incluido algunas acciones aplicadas que sirven de ejemplo para este trabajo. Los resultados se estructuraron en dos hallazgos fundamentales que sirvieron para una reflexión sobre la situación actual del estudiantado en relación a las acciones desarrolladas y como puntales para el mejoramiento de la didáctica de la lectoescritura en la carrera. De manera que se tabularon los resultados de las acciones emprendidas para la lectura y la escritura, siguiendo los parámetros descritos.

\section{Hallazgo I: Bajos niveles de competencia lectora en textos de género biografía.}

El análisis de la competencia lectora -acciones I a 3 - indica niveles bajos respecto a lo esperado en cada una de las acciones realizadas (Figura I). Considerando que el promedio de acierto en las respuestas corresponde a un rango mayor del 7o\%, se observan desaciertos que limitan los resultados positivos. De las acciones previstas para cada uno de los textos biográficos, las mayores dificultades tuvieron que ver con las acciones 2 y 3 , relacionadas con la identificación de la psicología de la persona y comportamientos, así como las razones a esos comportamientos y con el análisis de la interdependencia que existe entre las partes y el todo de los textos, así como con la valoración de los significados que se expresan en el texto biográfico. Realmente las dificultades estuvieron en el reconocimiento de rasgos del carácter de las personas, en los cuales se expresan sus relaciones con determinadas circunstancias y facetas de la realidad, así como el no recono- 
cimiento de argumentos y relaciones de causa-consecuencia, y de las relaciones entre los recursos movilizados para hacer significativo los conceptos o ideas claves en los textos. Estas dificultades están relacionadas, de forma general, con el pobre conocimiento que poseen los estudiantes con formas de trabajo con textos narrativos.

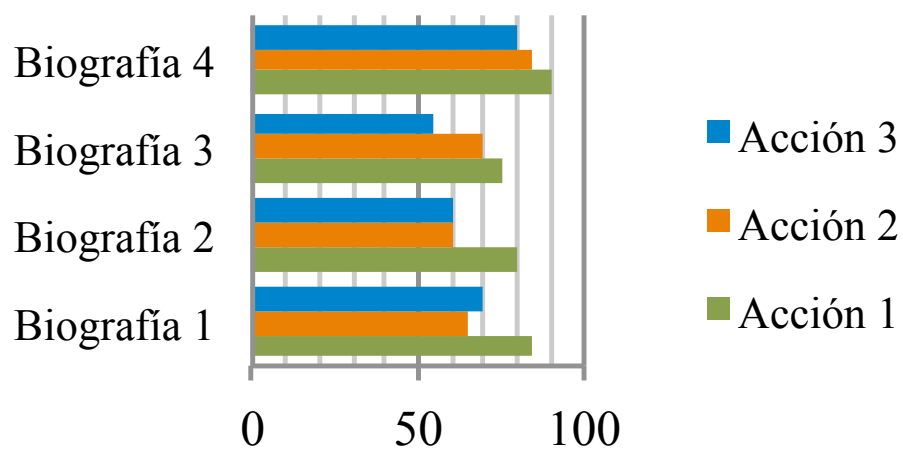

Figura I. Acierto en respuestas en las acciones de comprensión del texto biográfico

Hallazgo 2: El nivel de la competencia escritural es el resultado de una baja competencia lectora.

Los niveles de la competencia escritural - acciones 4 a 7 - indicaron valores de logro muy bajos. Por un lado los resultados de las acciones independientes mostraron dificultades en todas las acciones (Figura 2).

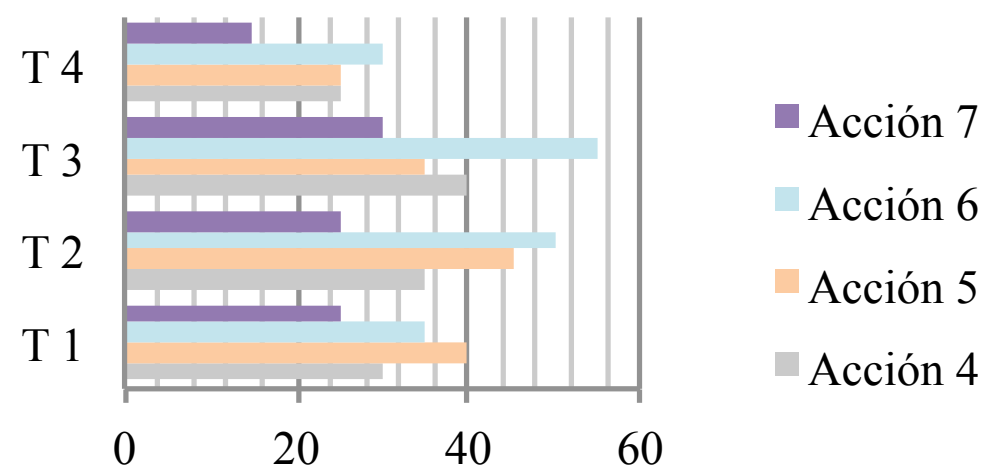

Fig. 2. Error en respuestas en la escritura

Considerando que un nivel adecuado corresponde al rango de $70-85 \%$, y alto a un rango mayor de 85 a Ioo\%, se observa que muchos estudiantes aún están en un rango bajo (Figura 3). 


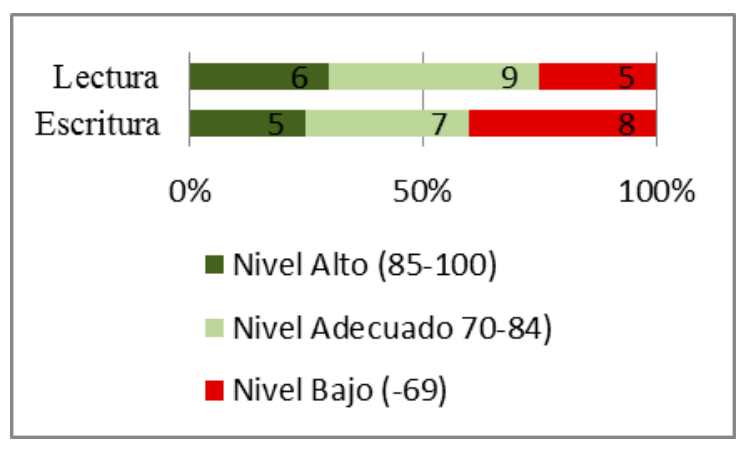

Fig. 3. Error en respuestas en la escritura

El cálculo del coeficiente de correlación (r) entre lectura y escritura de los 20 estudiantes arrojó los siguientes datos. La covarianza, que en este ejemplo es de 64.67 y el coeficiente de correlación de Pearson que en este caso es de o.87or3 indica una importante correlación entre las dos variables. Si elevamos al cuadrado el coeficiente de correlación obtendremos el coeficiente de determinación $(\mathrm{r} 2={ }=0.757$ I26) que nos indica que el $75 \cdot 7 \%$ de la variabilidad en el resultado de la escritura se explica por los resultados de la lectura, los que evidencian el estado de competencia lectora en los estudiantes. Ello significa que si se elevan los niveles de lectura podrían elevarse también los niveles de escritura, poniendo de manifiesto la interdependencia lectura-escritura.

\section{Hallazgo 2.I: Baja calidad del posicionamiento de los estudiantes frente a los} textos.

Este aspecto constituyó un punto neurálgico dentro de la experiencia. Una vez seleccionadas las ideas o conceptos claves, los estudiantes debían exponer criterios, opiniones y tomar posiciones en pequeños escritos, teniendo en cuenta que debía ser en lengua extranjera. Los resultados sobre el posicionamiento crítico (acción 7) revelaron un número significativo de dificultades que estuvieron entre el ${ }_{5} 5 \%$ y el $30 \%$ de errores en los textos biográficos seleccionados (Figura 4).

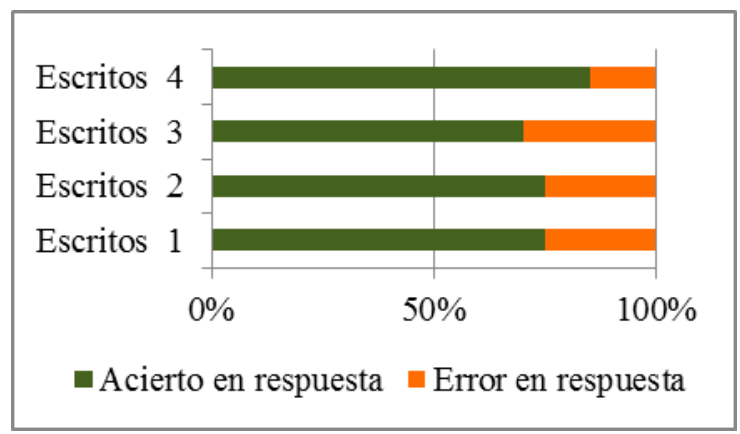

Figura 4. Escritura: posicionamiento crítico 
Un ejemplo de estos resultados sobre la biografía de Gertrudis Gómez de Avellaneda se expone en la tabla 2.

Como se aprecia, en el ejemplo escrito el estudiante intenta situarse como sujeto discursivo que evalúa dilemas morales e ideas y expone opiniones propias. En este sentido, procuramos indagar cuáles son los recursos utilizados por los estudiantes al expresar sus opiniones y cómo manejan otras voces, otras opiniones (inclusión de procedimientos de citas o referencias) y cuál es la calidad de las mismas. Se asume el hecho de que la cita o referencia a otros legitima la opinión expresada y refuerza la argumentación.

Tabla 2. Resultado de opinión sobre ideas de la biografía.

Her novel Dos mujeres (1842-1843) exposed women's social conditions under patriarchy, anticipating feminist awareness in a changing world.

Conceptos claves: Patriarchy, Feminist awareness.
"One of the things I admire about La Avellaneda is that she strongly attack the society governed by men. Women had no rights to participate in social life, to establish the same status as for men. In the time in which La Avellaneda lived, women were denied the legal and political rights, even the rights for intellectual development. I think women, as social beings, should have complete political, economic and social equality with men, and should be treated with respect and love and not as a mere housekeeper".

In an age of strict gender roles, Gómez de Avellaneda dared to step out of traditional roles: she married twice and maintained a fierce independence in a male-dominated society.

Conceptos claves: independence in a maledominated society.

Her anti-slavery novel $S a b$, which predates Uncle Tom's Cabin by more than a decade, was banned in Cuba-considered scandalous because of its abolitionist outlook, and its treatment of interracial love and societal conflict.

Conceptos claves: anti-slavery, abolitionist outlook, interracial love, societal conflict.
"In the time she lived she fought for her fully independence from a male-dominated society. She showed her courage and indomitable nature, even when she was attacked by her ideas about women's independence. Known as 'Tula' or 'La Peregrina', she was a great defender of the social role of women".

"She was a precursor of anti-slavery novels and had an abolitionist outlook. Her attitude and expectation for women's independence allowed to tackle many societal conflicts prevailed in the time she lived. In my opinion she wanted to be something so much worthier than the doll in the doll's house, as Bella said in Dickens's novel Our Mutual Friend". 
Tanto en el ejemplo como en la generalidad de los escritos de la muestra, es posible identificar el pobre uso de recursos de citas y referencias. Las opiniones de los estudiantes aparecen como extensiones de las ideas que aparecen en los textos biográficos, el uso de verbos se circunscriben a tipos 'think' 'in my opinion', utilizados en oraciones que incluyen atributos a favor de la posición de la figura en el texto y que sirven como extensión de lo dicho. Si bien hay intentos de posicionamiento al mostrar cierto acuerdo con el comportamiento o creencias de las figuras en los textos biográficos, son escasos los ejemplos que remitan a valoraciones de las causas de esos comportamientos o dilemas morales en los que se vieron envueltos esas figuras. En suma, los escritos de los estudiantes se ven contaminados por apreciaciones personales u opiniones que no llegan a estar lo suficientemente fundamentadas.

Los dos hallazgos que se muestran en este trabajo acentúan la necesidad de emprender una didáctica de la lectoescritura, de manera que se revelen de forma explícita las conexiones e interdependencias entre ambos, así como la presencia de procesos interrelacionados que utilizan un sustrato de conocimiento común, lo que facilita el proceso de comprensión y producción a través del proceso de deconstrucción del género.

\section{Discusión y conclusiones}

Los resultados de este trabajo evidencian algunas de las dificultades que muestran los estudiantes de lenguas extranjeras en la comprensión de textos biográficos y en la competencia escritural de orientación argumentativa.

La comprensión de lectura requiere de los estudiantes habilidades que van más allá del simple reconocimiento y dominio de estructuras lingüísticas que aparecen en los textos. La comprensión es un proceso de instrumentación intelectual que precisa la puesta en evidencia de relaciones o la creación de nuevos nexos; es un proceso activo de aprehensión y personalización del significado. Estas significaciones textuales sólo emerge cuando se establecen múltiples relaciones entre el texto y el contexto, el texto y otros textos y dentro de las partes internas del texto (Hernández, 20IO).

Ello conduce a considerar la práctica de lectura que se desarrolla en el aula, bien está carente de este tipo de actividades o no cubre una amplia gama de géneros textuales que contribuyan a la conformación de esquemas discursivos en los estudiantes y a la generación de significados propios sobre el contenido del texto, el contexto, el intertexto, etc. Los estudiantes, por su parte, no han desarrollado estrategias para enfrentar tareas de alta complejidad cognitiva, lingüística o socio-cultural. Así, las estrategias para la lectura que manejan son las que ponen en marcha, de forma errónea, cuando enfrentan situaciones que requieren de valoración o posicionamiento y sitúan al estudiante como sujeto con voz propia. No obstante, es necesario alertar que aun cuando la apropiación del lenguaje y las ideas ajenas sea algo cuestionable para muchos, esto es un indicador que refleja el inicio del desarrollo intelectual y cognitivo de los estudiantes. 
Es lógico pensar que mientras se continúe realizando prácticas de lectura en la que se privilegia el reconocimiento y la repetición de ideas de otros, los estudiantes continuarán "enmascarándose, es decir, tomando prestadas las voces de otros" (Castro y Sánchez, 2OI3: 5OI). Ello implica la necesaria tarea de sistematizar la lectura crítica, la identificación de argumentos y supuestos, el reconocimiento de relaciones e interrelaciones dentro del texto, la estimación del valor del discurso, de los significados subyacentes y de la ideología implícita, la identificación de elementos constitutivos de los géneros de estudio. También se hace necesario trabajar en el desmontaje o deconstrucción de géneros prototípicos para favorecer la escritura y, en este sentido, escribir sobre posiciones e ideas de otros, construir significados propios, crear posturas interpretativas y valorativas propias, todo lo cual conformará el necesario incentivo para el desarrollo de competencias en lengua extranjera.

Los problemas que se generaron en las acciones que realizaron los estudiantes de la muestra difícilmente se pueden solucionar si no se abordan desde el propio texto como herramienta discursiva o desde la propia lectoescritura como engranaje de comprensiónconstrucción. De hecho, "la construcción de significado a partir de la interacción entre el texto y el lector ha de enmarcarse en las circunstancias de lectura, algunas de las cuales suelen estar bastante convencionalizadas conformando géneros discursivos" (Carlino, 2008: 49). En efecto, esto constituye una pauta didáctica que se debe promover en las aulas de lenguas.

La comprensión de un texto requiere del dominio de los elementos que conforman el todo y sus partes. Como se mostró en la acción 3 sobre la organización hermenéutica del texto, los estudiantes aún carecen de estrategias para comprender el funcionamiento de las partes para acceder al significado del texto como un todo, expresadas de forma explícita o implícita. En este sentido, conforme a la teoría de la actividad de Leontiev (I98I), las acciones y operaciones inherentes a la actividad lectora se hacen significativas en relación con el todo. Pero solo la sistematización de esas acciones y operaciones posibilitarán la consolidación y el afianzamiento de determinadas formas de actuar de los estudiantes, de conocimientos y procedimientos para transformar el objeto. La particularidad de esos elementos consiste en que su formación solo es posible en el trabajo con el objeto discursivo, como es el caso que nos ocupa sobre el texto biográfico. Así, el estudiante debía apropiarse de procedimientos para acceder a los significados implícitos, a las partes no dichas para construir significados propios.

Es necesario destacar también los logros alcanzados en la experiencia. Las acciones descritas en este trabajo contribuyeron a la concientización de formas de pensar y actuar frente a un texto. También contribuyeron a que los estudiantes se dieran cuenta de sus puntos débiles en la lectura y escritura en lengua extranjera, fundamentalmente los relacionados con la comprensión/evaluación de significados implícitos y con la construcción de significados propios, así como con el desarrollo de estrategias de posicionamiento. 
En base a los resultados obtenidos y las formas de actuar de los estudiantes frente a los textos de estudio, fue posible construir un cuerpo de ideas que contribuirán al perfeccionamiento de la labor pedagógica en la carrera Lenguas Extranjeras. Así, al referirnos a la relación entre la comprensión lectora y la escritura, se asume el supuesto que si no se comprende el texto no se puede escribir sobre él. Así, cuando se analizan las acciones desarrolladas se observa que los aciertos en respuestas de comprensión lectora corresponden, en su mayoría, con aciertos en la escritura. De esta forma se identificaron estudiantes con niveles altos, niveles adecuados y niveles bajos, con niveles de competencia escritural deficitarios.

A partir del análisis correlacional lectura-escritura es posible proporcionar algunas pautas que deberían tenerse en cuenta para el trabajo en el aula de lenguas. Las actividades de lectura deben promover prácticas no solo de deconstrucción estructural de géneros textuales, sino también de resemantización de significados implícitos en los textos, que coadyuven al desarrollo del pensamiento crítico de los estudiantes. La enseñanza de la comprensión lectora no significa un acomodamiento de los textos a un listado de acciones o ejercicios. Por el contrario, cada texto requiere de un sistema de acciones propias en correspondencia con los tipos de relaciones que se establecen, los significados que se desarrollan y las formas en que estos se plasman en el texto.

La producción escrita, por su parte, es un proceso que exige de los estudiantes un mayor esfuerzo cognitivo, mayor disposición y dominio lingüístico. Su didáctica se sostiene, entre otros, en una "perspectiva significativa, articulada, socioconstructivista, crítica y explícita de la enseñanza y el aprendizaje de los géneros discursivos” (Navarro, 20I9: 23). Esta perspectiva didáctica deberá atender, además, al progreso gradual del conocimiento de los estudiantes acerca de las características formales de tipologías de textos diversos, lo que constituirá la esencia del trabajo didáctico. 


\section{Referencias bibliográficas}

-Arizpe, E. y Styles, M. (2002). ¿Cómo se lee una imagen? El desarrollo de la capacidad visual y la lectura mediante libros ilustrados. Lectura y Vida, Revista Latinoamericana de Lectura, 23 (3), I7-28. Recuperado el 4 de mayo de 2017 desde www.lecturayvida.fahce.unlp. edu.ar/numeros/a23n3/23_o3_Arizpe.pdf

- Avendaño de Barón, G.S. (20i6). La lectura crítica en Educación Básica Secundaria y Media: la voz de los docentes. Cuadernos de Lingüistica Hispánica, (28), 207-232. doi: http:// dx.doi.org/Io.I9053/or2I053X.49I6. Recuperado el I4 de marzo de 2019 desde https:// dialnet.unirioja.es/descarga/articulo/5603097.pdf

- Bazán, A., Ferrari, A., Castro, G., Delgado, U. y Hernández, C. (20i6).La autobiografía como aprendizaje esperado y comportamiento lingüístico en niños rurales mexicanos. Revista Argentina de Ciencias del Comportamiento, 8 (3), 6I-7I. Recuperado el I4 de marzo de 2OI9 desde https://dialnet.unirioja.es/descarga/articulo/5775368.pdf

- Bruner, J.S. (1994). The narrative construction of "reality". En M. Arnmaniti y D.N. Stem (Eds.), Psychoanalisis and development (pp. I5-38). New York: New York University Press.

- Bruner, J.S. (I995). Actos de significado. Más allá de la revolución cognitiva. Madrid: Alianza.

- Carlino, P. (2008). Didáctica de la lectura en la universidad. Ámbito de Encuentros, 2 (I), 47-67. Recuperado el 22 de abril de 2015 desde http://www.aacademica.org/paula.carlino/ 67

- Cassany, D. (2006). Taller de textos. Leer, escribir y comentar en el aula. Barcelona: Paidós.

- Cassany, D. (2008). Metodología para trabajar con géneros discursivos. Lenguajes de Especialidad y terminología: Didáctica y comunicación en ámbitos de especialidad. Euskara Institutua, Leioa.

- Castro, M.C. y Sánchez, M. (2OI3). La expresión de opinión en textos académicos escritos por estudiantes universitarios. Revista Mexicana de Investigación Educativa, I8 (57), 4835o6. Recuperado el 3 de julio de 2016 desde https://dialnet.unirioja.es/descarga/articulo/5345o69.pdf

- Dolz, J. y Gagnon, R. (20IO). El género textual, una herramienta didáctica para desarrollar el lenguaje oral y escrito. Lenguaje, $3^{8}$ (2), 497-527. Recuperado el 3 de julio de 2016 desde http://nexus.univalle.edu.co/index.php/lenguaje/article/view/49I7/7055

- Grabe y Kaplan (1998). Theory \& Practice of writing. Nueva York: Longman. 
- Hamui, S. (2OI4). La lectura como escritura: una mirada de Borges desde Derrida. Enunciación, I9 (I), I3I-I40. Recuperado el 3 de julio de 2016 desde https://dialnet.unirioja.es/ descarga/articulo/4995563.pdf

- Hernández, J. E. (2OI2). Criterios para la evaluación de la comprensión de textos como sistema de relaciones cognitivo-afectivas. Transformación, 8 (2), 24-36. Recuperado el I2 de septiembre de 2014 desde https://transformacion.reduc.edu.cu/index.php/transformacion/article/view/66

- Hernández, J. E. (2OIO). La instrumentación de la comprensión de textos en la escuela. Transformación, 6 (I), I7-27. Recuperado el 22 de enero de 2014 desde https://transformacion.reduc.edu.cu/index.php/transformacion/article/view/32

- Jarpa, M. (20I3). Una propuesta didáctica para el desarrollo de la escritura académica en estudiantes universitarios. Revista Iberoamericana de Evaluación Educativa, 6 (I), 29-48. Recuperado el 4 de abril de $2 \mathrm{OI}_{5}$ desde https://revistas.uam.es/index.php/riee/article/ view/3840

- Kintsch, W. (1998). Comprehension: a paradigm for cognition. Cambridge: Cambridge University Press.

- Leontiev, A. (I98I). Actividad, conciencia y personalidad. La Habana: Pueblo y Educación.

- Montealegre, R. (2004). La comprensión del texto: sentido y significado. Revista Latinoamericana de Psicología, 36 (2), 243-255. Recuperado el 22 de enero de 2014 desde https:// dialnet.unirioja.es/servlet/articulo? codigo $=$ II99o6o\&orden $=0 \& i n f o=l i n k$

- Moyano, E. (2007). Enseñanza de habilidades discursivas en español en contexto pre-universitario: Unaaproximación desdelaLSF. Revista Signos, 40(65), 573-6o8. Recuperado el 22 de enero de 20I4 desde https://www.scielo.cl/scielo.php?script=sci_arttext\&pid=So7r8o9342007000300009\&lng=es\&nrm=iso\&tlng=es

- Navarro (2OI9). Aportes para una didáctica de la escritura académica basada en géneros discursivos. D.E.L.T.A., 35(2), -32. http://dx.doi.org/IO.I590/I678-46oX2OI935O2OI

- Sánchez, S. (2OII). Relaciones intertextuales y competencia literaria en la obra narrativa de Fernando Alonso. OCNOS, 7, 7-22. Recuperado el 4 de abril de $205_{5}$ desde https://dialnet.unirioja.es/servlet/articulo? $\operatorname{codigo}=38 \mathrm{I} 53 \mathrm{I} 4$

- Santiago, A., Castillo, M. y Luz, D. (2007). Estrategias y enseñanza-aprendizaje de la lectura. Folios, 26, 27-38. Recuperado el 4 de abril de 20I5 desdehttps://dialnet.unirioja. es/servlet/articulo?codigo $=383205^{\mathrm{I}}$

- Sepúlveda, M.P. y Rivas, J.I. (2003). Voces para el cambio. Las biografías como estrategias 
de desarrollo profesional. En M.A. Santos y R. Beltrán (coords.), Conocimiento, ética y esperanza (pp. 367-38I). Málaga: Servicio de Publicaciones de la Universidad de Málaga.

- Serrano, S. y Madrid, A. (2007). Competencias de lectura crítica. Una propuesta para la reflexión y la práctica. Acción pedagógica, I6, 58-68. Recuperado el 4 de abril de 2015 desde https://dialnet.unirioja.es/servlet/articulo? $\operatorname{codigo}=2968602$

- Van Dijk, T. \& Kintsch, W. (1983). Strategies of discourse comprehension. New York: Academic Press. 